\title{
BOAS PRÁTICAS E BEM-ESTAR EM CAVALOS DE HIPISMO: OPORTUNIDADES DE MELHORIAS
}

\author{
Regiane Marques Sturn; Fernanda Tereza Lima²; Andrea R. Bueno Ribeiro ${ }^{3}$ \\ ${ }^{1}$ Mestre pelo Programa de Mestrado em Saúde Ambiental do Complexo Educacional \\ das Faculdades Metropolitanas Unidas - FMU. E-mail: regiane.sturn@fmu.br \\ ${ }^{2}$ Mestre pelo Programa de Mestrado em Saúde Ambiental do Complexo Educacional \\ das Faculdades Metropolitanas Unidas - FMU. \\ ${ }^{3}$ Profa. Dra. do Programa de Mestrado em Saúde Ambiental do Complexo \\ Educacional das Faculdades Metropolitanas Unidas - FMU.
}

Recebido em: 06/04/2018 - Aprovado em: 10/06/2018 - Publicado em: 20/06/2018 DOI: 10.18677/EnciBio 2018A20

\begin{abstract}
RESUMO
$\mathrm{Na}$ ciência, o estado de bem-estar de um animal é muitas vezes relacionado com o resultado dos efeitos das ações dos seres humanos, sendo esta voltada para 0 conhecimento das necessidades dos mesmos. Assim sendo, o animal que conseguir satisfazer suas necessidades biológicas terá maior grau de bem-estar e, de forma contrária, quando não atendidas, respostas fisiológicas e comportamentais são desencadeadas na tentativa de adaptação, mantendo sua homeostasia. Quando o equilíbrio não se estabelece, um estado permanente de estresse se mantém. Neste contexto, a avaliação do bem-estar nos equinos usados no hipismo, é importante, uma vez que esses são submetidos a ambientes e condições de manejo que não atendem na maior parte do tempo as necessidades da espécie. Sendo assim, o objetivo deste trabalho foi, por meio de uma revisão de literatura, evidenciar as necessidades e os principais desafios relacionados ao bem-estar dos cavalos de hipismo.
\end{abstract}

PALAVRAS-CHAVE: Adaptação. Comportamento. Equinos. Estereotipias. Manejo. Saúde.

\section{GOOD PRACTICES AND WELFARE OF SPORT HORSES: OPPORTUNITIES FOR IMPROVEMENTS}

\begin{abstract}
In science, animal's welfare condition is often related to the result of the human actions towards them, and it is focused on the understanding of their needs. Therefore, the animal that is able to satisfy its biological needs will have higher welfare level. Therefore, if it is not, there is a lack of satisfaction, triggering a permanent condition of stress. Physiological and behavioral responses will occur in the attempt of animal's body to adapt to that environment, maintaining its homeostasis. In this context, since the athlete equines are often kept in environments and submitted to management conditions that do not meet their needs, welfare evaluation is a major issue. Thus, the objective of this study is to understand equines needs, to identify key issues and alternative solutions related to the well-being of sport horses.
\end{abstract}

KEYWORDS: Adaptation. Behavior. Equine. Stereotypies. Handling. Health. ENCICLOPÉDIA BIOSFERA, Centro Científico Conhecer - Goiânia, v.15 n.27; p. 208 


\section{INTRODUÇÃO}

A definição do termo "bem-estar" não é simples. Broom (1986) definiu-o como "o estado de um indivíduo relacionado às suas tentativas de se adaptar ao ambiente". Já Malinowski (2004) considerou essa ciência como o estudo das reações psicofisiológicas dos animais ante aos estímulos externos gerados pelo homem, para identificar prováveis circunstâncias de implicações na condição de vida, podendo, assim, propor medidas para eliminar ou, ao menos, minimizar tais condições. Complementando, Broom e Molento (2004) atrelaram ao termo os conceitos de liberdade, felicidade, sofrimento, medo, tédio, ansiedade, dor, adaptação, competição, entre outros.

As preocupações sobre o bem-estar animal tiveram início por volta de 1800 , quando filósofos, como Voltaire e Jeremy Bentham começaram a questionar a prática da vivissecção. Todavia apenas no início do século XX as questões sobre a forma de manejo e criação dos animais de produção começaram a ser debatidas mais profundamente (BROOM, 2003). O marco inicial para discussões mais aprofundadas ocorreu em 1964, com a publicação do livro Animal machines de Ruth Harrison, que denunciava o tratamento dispensado aos animais de produção. Como resultado, no ano seguinte, a publicação do relatório de Brambell, comitê formado pelo governo britâncico por especialistas de diversas áreas, avaliou tecnicamente as questões levantadas pela autora e assim, pela primeira vez documentou-se que o bem-estar dos animais de produção estava comprometido e que os sistemas de criação deveriam estar alicerçados em condições relacionadas à necessidade destes.

Assim, em 1993 foram definidas pela Farm Animal Welfare Council as "Cinco Liberdades", que evidenciava que os animais deveriam ser livres de fome e sede; de dor, lesões e doenças; de medo e de estresse; de desconforto; e poder expressar seu comportamento natural (FAWC, 2009). As "Cinco Liberdades", segundo Broom (1999), permitem uma avaliação qualitativa do estado de bem-estar dos animais, onde são utilizados parâmetros que variam de "muito bom" à "muito pobre", sendo considerado algo que não se pode oferecer ao animal e sim algo intrínseco a ele, em um determinado momento, podendo-se atuar na tentativa de melhorá-lo. Nesse contexto, quando suas necessidades não são atendidas, o animal entra em um estado de estresse, que dependendo de sua severidade e tempo, influenciará de forma mais ou menos importante seu grau de bem-estar. Quando comprometido, esse estado pode resultar em reduzida expectativa de vida; redução no crescimento, produção ou reprodução; lesões corporais e doença; distúrbios comportamentais e opressão da atividade natural; imunossupressão; alteração do processo fisiológico normal e do desenvolvimento anatômico entre outros (BROOM; JOHNSON, 1993).

Normalmente equinos destinados ao esporte ou sob trabalho intenso são estabulados. A privação da liberdade, pastejo e comportamento social, o nível da movimentação física e a diminuição da ingestão de volumosos são fatores considerados estressores, prejudicando o bem-estar desses animais (HOUPT; MCDONNELL, 1993; MCGREEVY, 2004). Cortez e Silva (2007) definiram estresse como uma reação do organismo que tem por função a adaptação a um estímulo, podendo ser considerado um estado de resistência. Sendo assim, parâmetros fisiológicos e comportamentais de estresse podem ser usados como indicadores de bem-estar (MOBERG, 1987; SNOWDON, 1999; MOSTL; PALME 2002). 


\section{Evolução e comportamento social dos equinos}

A expressão do comportamento de um equino é resultado de diversos fatores, dentre os quais pode-se destacar o sistema de criação, o número e densidade de animais no grupo manejado e a qualidade do ambiente físico em que o animal é criado, além da característica comportamental do próprio indivíduo (LEWIS, 2000).

Os equinos são mamíferos, ungulados, monogástricos, pastejadores, que selecionam o seu alimento, sendo este predominantemente de folhas, colmos e brotos (SALTER; HUDSON, 1979; ELLIS; HILL, 2005). São animais velozes, podendo atingir até $60 \mathrm{~km} / \mathrm{h}$ (PESSOA, 2006), habilidade importante desenvolvida durante a evolução da espécie, uma vez que dependiam desta para escapar de seus predadores. Segundo Boyd (1991) e Smythe (1990), os cavalos selvagens gastam a maior parte do seu tempo pastando, cerca de 60 a $70 \%$, e o restante do tempo é preenchido observando outros animais, brincando, acariciando-se uns aos outros ou andando à procura de novas áreas de pastagem.

Segundo Grandin e Johnson (2010) os equinos também possuem visão muito aguçada, conseguindo identificar rapidamente a presença de um predador. São animais inteligentes, e possuem grande ligação social com seu tutor ou tratador. O sentimento de medo, a raiva e o pânico nos cavalos são resultado de lembranças adquiridas por condições que sofreram, e podem ser identificados por sinais comportamentais simples, como o balançar mais brusco da cauda, refugo, raspar o chão com os pés, posicionamento das orelhas e pescoço, entre outros.

São animais essencialmente sociais, que vivem em grupos matriarcais e que usam uma elaborada linguagem corporal para se comunicar uns com os outros e quando forçados a tomarem decisões, podem realizar ações prejudiciais à sua sobrevivência para escapar (ROBERTS, 2002; MILLS, 2005) A espécie equina apresenta uma complexa organização social, onde os indivíduos são dependentes de outros da mesma espécie, e para se adaptar a condições de vida isolada podem apresentar dificuldade (CINTRA, 2014). Todavia, nos sistemas atuais de criação, a área ocupada, o tempo de pastejo e os grupos sociais foram alterados de forma importante, e os animais permanecem quase que exclusivamente estabulados, confinado em pequenas baias onde vivem a maior parte do tempo (REZENDE et al., 2006á; GARCIA et al., 2010). Por essa inversão de atividades, doenças e comportamentos anormais têm sido observados com maior frequência nesses animais, sendo necessário proporcionar instalações e manejo que possibilitem melhor equilíbrio físico e mental a estes (CINTRA, 2014).

O confinamento interfere no sistema imunológico dos animais submetidos a essas condições, deixando-os mais vulneráveis devido ao estresse. Logo, esses animais ficam mais suscetíveis a doenças (BIRD, 2004; CINTRA, 2014) e cavalos treinados de forma intensa podem apresentar uma maior prevalência e gravidade de lesões (MURRAY et al., 1989). Alguns autores já ressaltavam que é comum em equinos que são cerceados a ambientes fechados adquirirem problemas respiratórios, devido à inalação de poeira ou amônia. Isso ocorre quando ficam em baias com baixa ventilação, onde a concentração de amônia é alta, independente do tipo de cama presente na baia (CURTIS et al.,1996).

Outros problemas que ocorrem comumente estão relacionados à nutrição. Uma dieta com maior proporção de concentrado, manejo nutricional normalmente utilizado e que não corresponde ao tipo de dieta que o sistema digestivo do cavalo foi adaptado, pode ocasionar cólicas, redução de apetite, má condição corporal, diarreia crônica e problemas dentários (MURRAY et al., 1989; CASEY, 2007; BURN ENCICLOPÉDIA BIOSFERA, Centro Científico Conhecer - Goiânia, v. 15 n.27; p. 210 
et al., 2010; MCGOWAN et al., 2010;). Além disso, a dieta pobre em volumoso está associada à apresentação de comportamentos estereotipados e problemas de saúde, como cólicas por impactação, em cavalos que ingerem a cama, e úlceras gástricas (THORNE et al., 2005). Esta última causa dor e perda da condição corporal, sendo também um dos fatores predisponentes no desenvolvimento de movimentos repetitivos como a aerofagia pertinente a intensa dor estomacal (CASEY, 2007). Segundo Bird (2004), os cavalos desenvolvem tais comportamentos na intenção de estimular a secreção de endorfinas que acalmam o cavalo na tentativa de fuga.

Então, para prevenir o desenvolvimento de estereotipias o ideal é proporcionar um ambiente e manejo alimentar mais próximo ao natural para o cavalo, permitindo interações sociais e possibilidade de realização de atividades físicas (REZENDE et al., 2006b;COOPER; MCGREEVY, 2007; PARKER, et al., 2008).

\section{O bem-estar de cavalos no esporte}

Devido as suas características físicas, o cavalo possui uma predisposição natural para uso no esporte sendo utilizado em diversas modalidades desportivas, como corridas, salto de obstáculos, adestramento, horseball (junção de basquete e futebol sobre os cavalos), entre outros. No entanto, o uso de cavalos como "ferramentas de competição" frequentemente resultam em problemas que influenciam direta ou indiretamente, não apenas seu bem-estar mas também seu desempenho esportivo (SILVA, 2010; BARTOLOMÉ; COCKRAM, 2016).

Equinos destinados ao esporte ou sob trabalho intenso são estabulados em baias de tamanho restrito, permanecendo por um longo período de tempo isolados, em ócio, com períodos curtos de alimentação, que geralmente é fornecida para consumo rápido no cocho. A restrição de pastejo e de convívio social, a intensidade da atividade física e a baixa ingestão de volumosos são fatores considerados estressores, e prejudicam o bem-estar desses animais (HOUPT; MCDONNELL, 1993; MCGREEVY, 2004).

Equinos criados em ambientes e em condições estressantes têm maior probabilidade de desenvolver doenças que acarretam, além de sofrimento, redução de desempenho, aumento dos custos de sua criação e o aparecimento de vícios e alterações comportamentais que comprometem seu bem-estar. Sendo assim, a avaliação e a melhoria das condições de manejo e criação dos equinos atletas tornam-se um fator importante para garantia de sua saúde e maior bem-estar animal (SALTER; HUDSON, 1979; MARCELLA, 1988; ALEXANDER; IRVINE, 1998; ELLIS, HILL, 2005; LEAL, 2007).

A avaliação do bem-estar requer uma abordagem multidisciplinar devido à complexidade dos processos adaptativos os quais envolvem a sanidade, as variáveis fisiológicas, a produtividade, as características comportamentais e as opções dos animais pelas variedades constituintes do ambiente que os rodeiam (BROOM, 1991; MENCH, 1993; ZANELLA, 1996).

O bem-estar está intimamente relacionado à ausência ou à ocorrência de uma resposta fisiológica de estresse (BARNETT; HEMSWORTH, 1990), pois o organismo animal em suas tentativas de adaptação, em face aos vários estímulos do meio ambiente e sob os esforços para satisfazer suas necessidades, apresentará respostas físicas e fisiológicas específicas, como alterações nas concentrações de hormônios do eixo hipotalâmico, nas funções cardio-respiratórias, e de metabólitos séricos. 
Equinos que participam de competições estão expostos a fatores estressores diferentes: dificuldade da competição, transporte, exames veterinários e proximidade forçada com desconhecidos (FAZIO, et al., 2008; MCGREEVY et. al., 2005; MEDICA, et al., 2010; SCHMIDT et al., 2010; HALL, et al., 2013). A pressão decorrente desses estressores tem um impacto no desempenho e no bem-estar do cavalo. Alguns estudos demonstraram que equinos que participam de competições apresentam um aumento transitório de liberação de cortisol e aumento da frequência cardíaca (CRAVANA, et al, 2010; PEETERS et al., 2011; BECKER-BIRCK et al., 2013). Corroborando com esses autores, em estudo recente, Jastrzębska, et al. (2017) concluíram que os animais que participam de competições esportivas apresentaram aumento dos níveis de cortisol salivar.

\section{A saúde física dos cavalos de esporte}

As lesões locomotoras estão entre os principais motivos de ocorrência médica em equinos, afetando-os independentemente da sua idade, gênero, raça ou utilidade (ROSS; DYSON, 2003). A maioria dos agravos que ocorrem nos membros anteriores, pois são responsáveis por suportar maior proporção do peso do animal, cerca de $60 \%$, e nestes, iniciam $95 \%$ no carpo ou distalmente a este (STASHAK, 1998).

Os cavalos praticantes da modalidade salto, por exemplo, estão sujeitos a lesões nas diversas estruturas do aparelho locomotor (THOMASSIAN, 2005). Dentre elas a síndrome do navicular e abcessos de casco, fraturas da segunda falange ou falange média, osteoartrite do boleto, traumatismos espinhais e vertebrais. A dor de origem músculo-esquelética também pode ser causada devido à intensa atividade física que esses animais são expostos, resultando em claudicação que poderá, também, variar de acordo com o tipo de atividade a qual o animal é submetido (ROSS; DYSON, 2003).

As afecções respiratórias na espécie equina são consideradas a segunda causa da diminuição do rendimento, suspensão do treinamento e a ausência prematura das competições sendo somente vencidas pelas alterações musculoesqueléticas (KUSANO et al., 2008). Alguns autores relataram que cerca de $42 \%$ dos cavalos atletas que apresentavam queda no desempenho foram identificados com doenças respiratórias (DAVIDSON; MARTIN, 2003). Dentre as mais frequentes estão a doença pulmonar obstrutiva crônica (DPOC), hemorragia pulmonar induzida pelo exercício (HPIE) (GEOR,1995) e a disfunção neuromuscular chamada de deslocamento dorsal do palato mole (DDPM) (MORRIS; SEEHERMAN, 1990; HOLCOMBE et al., 1998).

Como regra geral, pode-se dizer que quanto maior a velocidade alcançada ou mais intenso o exercício, maior a proporção de cavalos afetados pela HPIE. Pascoe (1991) registrou em estudo uma ocorrência de 42 a $85 \%$ em equinos submetidos a exercícios de velocidade. Ainsworth e Biller (2000) registraram a prevalência da HPIE de 44 a $75 \%$ em cavalos de corrida, de $40 \%$ em cavalos de eventos de 3 dias e $11 \%$ nos de pólo. Em estudo realizado por Suma (2002) no Departamento de Veterinária nas Corridas do Jóquei Clube Brasileiro (JCB), em 6.601 endoscopias realizadas em cavalos Puro Sangue Inglês de diferentes sexos e idades no período de janeiro de 2002 a novembro de 2003, foram registradas 7.023 doenças (alguns animais apresentaram mais de uma) sendo $54,4 \%$ ocorrências de HPIE. Além das afecções respiratórias também podem adquirir doenças de origem infectocontagiosa como a influenza, o garrotilho e o mormo (ACHA; SZYFRES, 2003). 
Segundo Reef (2001), a terceira causa mais comum de intolerância ao exercício em equinos atletas de elite, atrás apenas das afecções músculoesqueléticas e respiratórias, são os problemas cardiovasculares. Estes geralmente são parte de um processo multifatorial, mas podem vir como única causa de declínio de desempenho. A modalidade salto do hipismo clássico, mescla salto e corrida, e gera necessidade de melhor condicionamento físico por parte dos equinos além da sobrecarga do sistema cardiovascular, assim, portanto, pode propiciar o desenvolvimento de arritmias (DINIZ et al, 2011). Já as doenças vasculares de episódios mais frequente estão associadas à trombose isquêmica, incluindo a laminite, arterite verminótica, vasculites (púrpura hemorrágica, imunomediada, arterites virais), a oclusão de veia decorrente a um coágulo e a laminite (BAYARS et al., 2003; WIEMER et al., 2005).

\section{Comprometimento gastrointestinal}

Outro fator importante a ser obervado são os relacionados à alimentação. As vantagens da domesticação estão relacionadas com a oferta de alimento e abrigo contra predadores. Já os custos foram, principalmente, a restrição de movimentos, falta de interação social e alimentação artificializada (GOODWIN, 2007). Como consequência dessa domesticação houve uma queda do tempo disponível para o cavalo pastar naturalmente, e que determinou alterações importantes no comportamento alimentar, aumentando assim a possibilidade de desorganizações gastrointestinais e bem-estar reduzido. Estas consequências são evidenciadas pela frequência de distúrbios comportamentais nesses animais (JOHNSON et al., 1998).

Em relação à alimentação, a simplificação da dieta embasada no atendimento principalmente das carências nutricionais prevalece nos sistemas de criação e trazem problemas de ordem diversas, pois não há reflexão sobre aspectos relacionados as formas de disponibilização dos alimentos e as necessidades comportamentais desses animais (DITTRICH, 2010). Cavalos que consomem em abundância o concentrado por refeição têm oscilações intensas de glicose e insulina plasmática, causando grande oscilação nos níveis energéticos, e por consequência em seu comportamento.

Somam-se, nessas condições, a maior ocorrência de desordens digestivas e metabólicas, como cólicas e ulcerações gástricas, as quais provocam uma sequência de variações no comportamento do animal devido a intensa dor (CAMPELO; PICCININ, 2008). O equino é muito sensível às variações ambientais e de manejo alimentar. A diminuição ou oscilação no nível da prática física, alterações nas condições de estabulação, privação de água, alterações súbitas na dieta, refeições ricas em concentrados, volumoso ou concentrado de má qualidade, ingestão excessivamente rápida da ração concentrada e a condução em viagens podem motivar a ocorrência de Síndrome Cólica (HILLYER et al., 2001).

A cólica equina é uma desordem do aparelho digestório que esta relacionada a diversos fatores, podendo ser intestinais ou gástricos, obstrutivos ou não, com ou sem estrangulamento vascular. As cólicas de origem estomacal ocorrem principalmente quando há mudanças na fermentação microbiana ou são resultado da perda de motilidade e o risco de cólica aumenta com a abundância de concentrado consumido (GONÇALVES et al., 2002; VERVUERT; COENEN, 2004).

Com relação ao tipo não cirúrgico de cólica há as cólicas por distensão que geralmente são causadas por excesso de grãos ou por gases gerados por alimentos fermentáveis (CARTER, 1987). Nessa categoria incluem-se ainda as cólicas espasmódicas, distensões gasosas e impactações com obstrução parcial. Já as 
alterações decorrentes do intestino delgado são relacionadas com a necessidade de cirurgia, principalmente as obstruções e lesões estrangulantes como o encarceramento (TURNER et al., 1984; ENGELBERT et al., 1993; VACHON; FISCHER, 1995; MAIR 2002).

Outra importante afecção causadora de desconforto abdominal em equinos são as úlceras gástricas. Essas são causadas por corrosão ácida (péptica) sobre a mucosa gástrica dos equinos, podendo ocorrer com animais de qualquer idade. Fatores como confinamento, estresse, jejum, alimentação, manejo, ritmo intenso de treinamento ou administração de alguns medicamentos, como drogas antiinflamatórias não esteroides, são elementos que predeterminam sua ocorrência. Alimentos utilizados na suplementação de animais de esporte predispõem-se a úlceras gástricas em equinos, principalmente relacionados à ácidos graxos voláteis (AGV) decorrentes da fermentação desses alimentos (PALMA-DALLA et al., 2007; RIBEIRO et al., 2016). Quando estes animais secretam ácido gástrico continuamente e não recebem o alimento de forma contínua, a mucosa do estômago fica exposta a estes ácidos. Isso ocorre, pois, a única proteção que a mucosa teria seria oriunda da produção de saliva, a qual é gerada em quantidade ideal apenas com a deglutição de uma dieta rica em fibras (SILVA, 2014).

\section{Necessidades e alterações comportamentais em cavalos de esporte}

Existem inúmeros parâmetros que podem ser identificados, registrados e comparados com o objetivo de quantificar a qualidade do bem-estar de um determinado grupo de animais (BROOM; MOLENTO, 2004), entre eles a demonstração de uma variedade de comportamentos normais, grau que comportamentos fortemente preferidos que são apresentados, tentativas comportamentais de adaptação, auto narcotização, grau de aversão comportamental e grau de supressão de comportamento normal (PULZ, 2013).

Segundo Broom e Molento (2004) à medida que a exposição a um fator estressante se mantém, pode haver alteração na quantidade ou tipo de comportamento anormal assumido pelo animal, afirmação esta que corrobora com a relação entre os comportamentos anormais e o confinamento.

As observações etológicas têm também grande relevância na análise do bemestar. Os costumes anormais, tais como os movimentos repetitivos, a automutilação, o canibalismo, a agressividade abundante e mesmo a apatia indicam condições relacionadas a bem-estar ruim, todavia os tratadores ou responsáveis não reconhecem esses comportamentos como problemas de saúde em seus cavalos (ZANELLA, 1995; BROOM; MOLENTO, 2004; VIEIRA, 2015).

Em alguns sistemas de criação de equinos, os animais são privados de expressar suas necessidades e comportamentos em ambiente natural por permanecerem confinados em pequenas baias por muito tempo. São privados de interação social com indivíduos da mesma espécie ocupam que pode afetar no seu comportamento (REZENDE et al., 2006a). Nessas situações, alguns comportamentos anormais podem ser observados em decorrência da frustração do animal por estar privado de manifestar certos comportamentos naturais em ambientes tão restritivos. Estudo realizado por Júnior (2015) identificou que animais que permaneciam estabulados por mais tempo apresentavam $49 \%$ mais comportamentos anormais do que aqueles que tinham um período maior no pasto do que na baia.

Considerando que os equinos necessitam de liberdade, mantê-los confinados, foi interessante no processo de domesticação, possibilitando um manejo em pastos, 
fator determinante para a criação. Porém, manter essa espécie presa por muitas horas, sem distrações e atividades, além de retirá-la de sua família ou bando, interferindo em seu instinto natural, pode levar esses animais a desenvolverem determinados vícios e comportamentos não naturais e estereotipias (KONIECZNIAK et al., 2014).

Estereotipias são definidas como comportamentos anormais invariáveis e repetitivos que não tem nenhuma função e são considerados indicadores potenciais de desordens fisiológicas com consequente diminuição do bem-estar dos equinos (WATERS et al., 2002). Este tipo de comportamento anormal normalmente se desenvolve em animais que enfrentam dificuldade de adaptação à determinado ambiente em que foram inseridos. Assim, além de indicar alteração psicológica, esta evidencia que a saúde do animal está comprometida (BROOM; KENNEDY, 1993).

A hipótese de que as estereotipias são consideradas indicadores de baixo grau de bem-estar e de ambientes empobrecidos, têm sido amplamente discutidas. Dentre as estereotipias, há comportamentos orais atípicos como a geofagia, a coprofagia e o ato de comer pedaços de cascas de árvore, cercas, cama da baia etc. (JOHNSON et al., 1998; WATERS et al., 2002; BOYD; DEIPER, 2005). Alguns exemplos de sequência de movimentos repetidos com pequena variação na forma e que são comportamentos facilmente reconhecidos por um observador são: roer madeira, aerofagia com ou sem apoio, fazer movimentos repetitivos de balanço também conhecidos como a "síndrome do urso", dentre outros (BROOM; KENNEDY, 1993). Já a aerofagia, ato de engolir ar, tem como principal causa o tédio que acomete cavalos estabulados. Este vício causa diversos problemas como cólicas gasosas, desgaste excessivo dos dentes e, ainda, perda de peso (LEWIS, 1985; MARCELLA, 1988).

Neste contexto, os comportamentos destrutivos são aqueles que provocam lesões nos animais, tais como agressividade, mordeduras e os vícios de sucção de orelha, cauda, flanco e vulva (ZANELLA, 1995; SOBESTIANSKY; ZANELLA, 2007). Alguns animais são agressivos somente em determinadas situações, como quando estão se alimentando, no manuseio da cabeça ou membros, durante o estro (cio), na baia ou quando são capturados no pasto (MARCELLA, 1988). A agressão pode ocorrer com pessoas ou com outros animais, porém entre equinos a agressividade é normalmente resultado da disputa de dominância, a qual determina qual animal será o primeiro a guiar o rebanho ou ter acesso ao alimento (HOGAN et al., 1988).

Outro fator de relevância que interfere no comportamento equino é o manejo alimentar. Cavalos estabulados recebem, geralmente, grandes quantidades de alimento concentrado e, quando a quantidade de alimento volumoso é oferecida de maneira insuficiente, os indicadores fisiológicos de saciedade podem não ser ativados de maneira satisfatória e os animais permanecem com alta motivação à procura de alimentos (MCGREEVY et al., 1995). O confinamento excessivo pode desencadear acentuadas mudanças comportamentais, que não são evidenciadas em cavalos mantidos em pastagens (MILLS, 2005).

Em estudo realizado por Johnson et al. (1998) foi observado que disponibilizar altas proporções de alimentos concentrados na dieta de equinos confinados, provoca 0 aumento da incidência de distúrbios orais de comportamento (comportamentos anormais), como lamber cocho/grade, morder grade/muro e comer a cama. Ribeiro et al. (2009) ao compararem o comportamento e distúrbios alimentares em equinos alimentados com dietas a base de concentrado com aqueles alimentados apenas com feno, constatou que esses últimos apresentaram menos tempo ocioso para adquirir distúrbios de comportamento por passarem mais 
tempo comendo. Fornecer alimentos volumosos aos animais, principalmente estabulados, é uma importante ferramenta para manter a saúde física e mental dos equinos, pois este é um tipo de alimento de ingestão lenta e que permite aos animais expressarem o seu comportamento alimentar mais similar possível ao dos animais em liberdade (NATIONAL RESEARCH COUNCIL, 2007).

Cavalos que participam de competições de alta performance podem apresentar comportamentos anormais em decorrência da frustração, definido como comportamento de conflito, indicando algum tipo de desconforto e hiperatividade desses animais. Tais comportamentos incluem levantar a cauda, puxar as rédeas, tremor de corpo e cabeça, recusa (por exemplo, parando na frente de um obstáculo), e muitas vezes são indicativos de estresse (MCGREEVY et al. 2005; MINERO et al., 2008; WILLIAMS; WARREN-SMITH, 2010; HALL et al., 2013; SMIET, et al., 2014; GÓRECKA-BRUZDA et al., 2015).

Estes podem ser provocados pelo uso métodos invasivos de treinamento, erros de treinamento e pressão excessiva em cavalos não ainda preparados (VON BORSTEL et al., 2009; FERLAZZO et al., 2012; BECKER-BIRCK et al., 2013; CHRISTENSEN et al., 2014).

Em estudo realizado por Jastrzębska et al. (2017), observou-se que os equinos que apresentaram mais erros durante as competições esportivas, exibiram também maior frequência de comportamento de conflito e aumento dos níveis de cortisol salivar. Viagens antes das competições também afetaram a frequência de comportamento de conflito e cortisol nos cavalos. Medica et al. (2010) relataram que os cavalos transportados tiveram maiores concentrações de cortisol pós-exercício do que os cavalos não transportados.

Além disso, animais que são expostos a intervalos longos entre uma rodada e outra da competição apresentam maior frequência de comportamento de conflito. Isso pode ser explicado pelo desvio da rotina diária: a competição difere do treinamento regular, onde o cavalo geralmente termina seu trabalho dentro de um tempo preditivo e nenhum outro esforço é exigido. Assim, o efeito da imprevisibilidade das exigências humanas, pode ser estressante um fator estressante por si só (KOOLHAAS, et al., 2011). Tais resultados evidenciam que o estresse relacionado ao esforço e ao transporte para competição, podem em conjunto com ambientes e instalações desconhecidas, interferir no desempenho esportivo do animal.

As instalações e o comportamento social têm grande importância para o bemestar dos equinos (DE OLIVEIRA et al., 2016). Cintra (2014) avalia que preservar ou, ao menos, respeitar os hábitos dos cavalos traz ao homem muito mais benefícios. Para isso, o ser humano deve estudar, compreender, entender e levar em consideração as inter-relações que há entre o equino e o meio ambiente e a forma com que o animal se expressa.

Neste contexto, o respeito do homem é de grande importância possibilitar a manutenção do comportamento social para essa espécie. Assim, deve-se planejar instalações e práticas de manejo e rotina que permitam a formação e manutenção dos vínculos sociais (DE OLIVEIRA, 2016).

\section{CONSIDERAÇÕES FINAIS}

Considerando a literatura consultada, cuidados em relação à estabulação, rotina, alimentação, doenças e lesões, fadiga, interação social e estresse devem ser priorizados para equinos utilizados em provas hípicas, de maneira que sentimentos e sensações positivas estejam presentes e evidenciados na rotina desses animais. 
Considerando que "bem-estar" é um conceito multidimensional, envolvendo emoções, reconhecimento das condições físicas e psicológicas de acordo com estilo de vida de qualquer animal, e que os equinos são seres sencientes, mudanças de atitudes devem ir além de apenas evitar o sofrimento, mas sim promover a melhoria do bem-estar e qualidade de vida desses animais.

\section{REFERÊNCIAS}

ACHA, Pedro N.; SZYFRES, Boris. Zoonosis y enfermedades transmisibles comunes al hombre ya los animales: Volumen I. Organización Panamericana de la Salud, 2003.

AINSWORTH, D. M.; BILLER, D.; REED, S.; BAYLY, W. Sistema respiratório. REED, SM; BAYLY, WM Medicina interna equina. Rio de Janeiro: Guanabara Koogan, p. 217-249, 2000.

ALEXANDER, S.; IRVINE, C. H. G. Stress in the racing horse: Coping vs not coping. Journal of equine science, v. 9, n. 3, p. 77-81, 1998. Disponível em:<https://doi.org/10.1294/jes.9.77>. doi: 10.1294/jes.9.77

BARNETT, J. L.; HEMSWORTH, P. H. The validity of physiological and behavioural measures of animal welfare. Applied Animal Behaviour Science, v. 25, n. 1-2, p. 177-187, 1990. Disponível em:<https://doi.org/10.1016/0168-1591(90)90079-S>. doi: 10.1016/01681591(90)90079-S

BARTOLOMÉ, E.; COCKRAM, M. S. Potential effects of stress on the performance of sport horses. Journal of Equine Veterinary Science, v. 40, p. 84-93, 2016. Disponível em:<https://doi.org/10.1016/j.jevs.2016.01.016>. doi: 10.1016/j.jevs.2016.01.016

BAYARS, T. D.; DAVIS, D.; DIVERS, T. J. Coagulation in the equine intensivecare patient. Clinical Techniques in Equine Practice., v. 2, p. 178-187, 2003. Disponível em:<https://doi.org/10.1016/S1534-7516(03)00014-3>. doi: 10.1016/S1534-7516(03)00014-3

BECKER-BIRCK, M.; SCHMIDT, A.; LASARZIK, J.; AURICH, J.; MÖSTL, E.; $\mathrm{AURICH}, \mathrm{C}$. Cortisol release and heart rate variability in sport horses participating in equestrian competitions. Journal of Veterinary Behavior: Clinical Applications and Research, v. 8, n. 2, p. 87-94, 2013. Disponível em:<https://doi.org/10.1016/j.jveb.2012.05.002>.

doi:

10.1016/j.jveb.2012.05.002

BIRD, J. Cuidado Natural del Caballo. Barcelona: Acanto, 2004, 206 p.

BOYD, L. E. The behaviour of Przewalski's horses and its importance to their management. Applied Animal Behaviour Science, v. 29, n. 1-4, p. 301-318, 1991. Disponível em:<https://doi.org/10.1016/0168-1591(91)90256-W>. doi: 10.1016/0168-1591(91)90256-W 
BOYD, L.; KEIPER, R. Behavioural ecology of feral horses. The domestic Horse, the Evolution, Development and Management of Its Behaviour. Cambridge University Press, Cambridge, p. 55-82, 2005.

BROOM, D. M. Animal welfare: concepts and measurement. Journal of animal science, v. 69, n. 10, p. 4167-4175, 1991.

BROOM, D. M. Animal Welfare: the concept and the issues. In: DOLINS, F. L. Attitudes to animals: views in animal welfare. Cambridge: Cambridge University Press, 1999. p. 129-142.

BROOM, D. M. Indicators of poor welfare. British veterinary journal, v. 142, n. 6, p. 524-526, 1986.

BROOM, D. M. The evolution of morality and religion. $1^{\text {st }}$ ed. Cambridge: Cambridge University Press, 2003. 272p.

BROOM, D. M.; JOHNSON, K.G. BROOM, Donald M.; JOHNSON, Ken G. Assessing welfare: Short-term responses. In: Stress and animal welfare. Springer, Dordrecht, 1993. p. 87-110.

BROOM, D. M.; KENNEDY, M. J. Stereotypies in horses: their relevance to welfare and causation. Equine Veterinary Education, v. 5, n. 3, p. 151-154, 1993. Disponível em:<https://doi.org/10.1111/j.2042-3292.1993.tb01026.x>. doi: 10.1111/j.2042-3292.1993.tb01026.x

BROOM, D. M.; MOLENTO, C. F. M. Bem-estar animal: conceitos e questões relacionadas - Revisão. Archives of Veterinary Science, v. 9, n. 2, 2004.

BURN, C. C.; DENNISON, T. L.; WHAY, H. R. Relationships between behaviour and health in working horses, donkeys, and mules in developing countries. Applied Animal Behaviour Science, v. 126, p. 109-118, 2010. Disponível em:<https://doi.org/10.1016/j.applanim.2010.06.007>. doi: 10.1016/j.applanim.2010.06.007

CAMPELO, J.; PICCININ, A. Cólica Equina. Revista Científica Eletrônica de Medicina Veterinária. Ano VI, n. 10, 2008

CARTER, G. K. Gastric diseases. In: ROBINSON, N. E. Current Therapy in Equine Medicine, v. 2, p. 41, 1987.

CASEY, R. A. Clinical problems associated with the intensive management of performance horses. In: The welfare of horses. Springer, Dordrecht, 2007. p. 19-44. Disponível em:<https://doi.org/10.1007/978-0-306-48215-1_2>. doi: 10.1007/978-0-306-48215-1_2 
CHRISTENSEN, J. W.; BEEKMANS, M.; VAN DALUM, M.; VanDierendonck, $M$. Effects of hyperflexion on acute stress responses in ridden dressage horses. Physiology \& behavior, v. 128, p. 39-45, 2014.

CINTRA, A. G. C. O CAVALO: Características, Manejo e Alimentação. $2^{\circ}$ Reimpressão. São Paulo: Roca, 2014.

COOPER, J.; MCGREEVY, P. Stereotypic behavior in the stabled horse: causes, effects and prevention without compromising horse welfare. In: The welfare of horses. Springer, Dordrecht, 2007. p. 99-124. Disponível em:<https://doi.org/10.1007/978-0-306-48215-1_5>. doi: 10.1007/978-0-30648215-1_5

CRAVANA, C., MEDICA, P.; PRESTOPINO, M.; FAZIO, E.; FERLAZZO, A. Effects of competitive and noncompetitive showjumping on total and free

iodothyronines, $\beta$-endorphin, ACTH and cortisol levels of horses. Equine

Veterinary Journal, v. 42, n. s38, p. 179-184, 2010. Disponível em:<https://doi.org/10.1111/j.2042-3306.2010.00264.x>. doi: 10.1111/j.20423306.2010.00264.x

CURTIS, L.; RAYMOND, S.; CLARKE, A. Dust and ammonia in horse stalls with different ventilation rates and bedding. Aerobiologia, v. 12, p. 239-247, 1996. Disponível em:<https://doi.org/10.1007/BF02248160>. doi: 10.1007/BF02248160

DAVIDSON, E. J.; MARTIN, B. B. Diagnosis of upper respiratory tract diseases in the performance horse. Veterinary Clinics of North America: Equine practice, v. 19, n. 1, p. 51-62, 2003.

DE OLIVEIRA, J. N.; PEREIRA, A. T.; NATH, J. L. INTERRELAÇÃO ENTRE AS INSTALAÇÕES, COMPORTAMENTO SOCIAL E SEUS EFEITOS SOBRE O BEM-ESTAR EM CAVALOS DOMÉSTICOS. Salão do Conhecimento, v. 2, n. 2, 2016.

DINIZ, M. P.; MICHIMA, L. E.; FERNANDES, W. R. Estudo eletrocardiográfico de equinos de salto sadios. Pesquisa Veterinária Brasileira, v. 31, n. 4, p. 355-361, 2011.

DITTRICH, J. R.; MELO, H. A.; AFONSO, A. M. C. F.; DITTRICH, R. L. Comportamento ingestivo de equinos e a relação com o aproveitamento das forragens e bem-estar dos animais. Revista Brasileira de Zootecnia, v. 39, p. 130-137, 2010.

ELLIS, A. D.; HILL, J. Nutritional physiology of the horse. Journal of Equine Veterinary Science, v. 25, n. 12, p. 524, 2005. Disponível 
em:<https://doi.org/10.1016/j.jevs.2005.11.002>.

doi:

10.1016/j.jevs.2005.11.002

ENGELBERT, T. A.; TATE, L. P.; BOWMAN, K. F; BRISTOL, D. G. Incarceration of the small intestine in the epiploic foramen report of 19 cases (1983-1992). Veterinary Surgery, v. 22, n. 1, p. 57-61, 1993. Disponível em:<https://doi.org/10.1111/j.1532-950X.1993.tb00370.x>.

doi: 10.1111/j.1532-950X.1993.tb00370.x

ERICKSON, H. A review of exercise-induced pulmonary hemorrhage and new concepts for prevention. Proc. Ann. Conv. Am. Assoc. Equine Pract, v. 46, p. 193-196, 2000.

FAWC (FARM ANIMAL WELFARE COUNCIL). Farm animal welfare in great britain: past, present and future. London: Press Statement, 2009. p. 1-59.

FAZIO, E.; MEDICA, P.; CRAVANA, C.; FERLAZZO, A. Effects of competition experience and transportation on the adrenocortical and thyroid responses of horses. The Veterinary Record, v. 163, n. 24, p. 713-716, 2008. Disponível em:<https://10.1136/vr.163.24.713>. doi: 10.1136/vr.163.24.713

FERLAZZO, A.; MEDICA, P.; CRAVANA, C.; FAZIO, E. Circulating $\beta-$ endorphin, adrenocorticotropin, and cortisol concentrations of horses before and after competitive show jumping with different fence heights. Journal of equine veterinary science, v. $32, n$. 11, p. 740-746, 2012. Disponível em:<https://doi.org/10.1016/j.jevs.2012.02.021>. 10.1016/j.jevs.2012.02.021

GARCIA, H. A. C.; FURTADO, C. E.; SONCIN, M. R. S. P.; WANDEMBRUCK, K. T., POLIZEL, V. P.; TORRECILHAS, J. A. Diferença comportamental entre Potros, machos e fêmeas, cruzados puro sangue Inglês e Mangalarga submetidos a inicio de cabrestamento e estabulagem. Revista da FZVA, v. 17, n. 2, 2010.

GEOR, R. J. Performance-limiting conditions of the lungs. The Horse. Diseases e Clinical Management, v. 1, p. 263-273. Philadelphia: Saunders, 1995.

GONÇALVES, S.; JULLIAND, V.; LEBLOND, A. Risk factors associated with colic in horses. Veterinary Research, v. 33, p. 641-652. 2002. Disponível em:<https://doi.org/10.1051/vetres:2002044>. doi: 10.1051/vetres:2002044

GOODWIN, D. Horse behaviour: evolution, domestication and feralisation. In: The welfare of horses. Springer, Dordrecht, 2007. p. 1-18. Disponível em:< https://doi.org/10.1007/978-0-306-48215-1_1>. doi: 10.1007/978-0-306-482151_1

GÓRECKA-BRUZDA, A.; KOSIŃSKA, I.; JAWORSKI, Z.; JEZIERSKI, T.; MURPHY, J. Conflict behavior in elite show jumping and dressage horses.

ENCICLOPÉDIA BIOSFERA, Centro Científico Conhecer - Goiânia, v.15 n.27; p. 2202018 
Journal of Veterinary Behavior: Clinical Applications and Research, v. 10, n. $2,2015 . \quad$ p. $137-146, \quad$ Disponível em:<https://doi.org/10.1016/j.jveb.2014.10.004>. doi: 10.1016/j.jveb.2014.10.004

GRANDIN, T.; JOHNSON, C. O bem estar dos animais: proposta de uma vida melhor para todos os bichos. São Paulo: Rocco, 2010.

HALL, C.; HUWS, N.; WHITE, C.; TAYLOR, E.; OWEN, H.; MCGREEVY, P. Assessment of ridden horse behavior. Journal of Veterinary Behavior: Clinical Applications and Research, v. 8, n. 2, p. 62-73, 2013. Disponível em:<https://doi.org/10.1016/j.jveb.2012.05.005>.

doi: 10.1016/j.jveb.2012.05.005

HILLYER, M. H.; TAYLOR, F. G. R.; FRENCH, N. P. A cross-sectional study of colic in horses on Thoroughbred training premises in the British Isles in 1997. Equine Veterinary Journal, v. 33, n. 4, p. 380-385, 2001. Disponível em:<https://doi.org/10.2746/042516401776249499>. doi: $10.2746 / 042516401776249499$

HOGAN, E. S.; HOUPT, K. A.; SWEENEY, K. The effect of enclosure size on social interactions and daily activity patterns of the captive Asiatic wild horse (Equus przewalskii). Applied Animal Behaviour Science, v. 21, n. 1-2, p. 147-168, 1988. Disponível em:<https://doi.org/10.1016/0168-1591(88)901050>. doi: 10.1016/0168-1591(88)90105-0

HOLCOMBE, S. J.; DERKSEN, F. J.; STICK, J. A.; ROBINSON, N. E. Effect of bilateral blockade of the pharyngeal branch of the vagus nerve on soft palate function in horses. American journal of veterinary research, v. 59, n. 4, p. 504-508, 1998.

HOUPT, K. A.; MCDONNELL, S. M. Equine Stereotypies. Comp. On Cont. Educ. Pract. Vet. v.15, n.9, p.1265-1271, 1993.

JASTRZĘBSKA, E.; WOLSKA, A.; MINERO, M.; OGŁUSZKA, M.; EARLEY, B.; WEJER, J.; GÓRECKA-BRUZDA, A. Conflict Behavior in Show Jumping Horses: A Field Study. Journal of Equine Veterinary Science, v. 57, p. 116121, 2017. Disponível em:<https://doi.org/10.1016/j.jevs.2017.07.009>. doi: 10.1016/j.jevs.2017.07.009

JOHNSON, K. G.; TYRRELL, J.; ROWE, J. B.; PETHICK, D. W. Behavioural changes in stabled horses given nontherapeutic levels of virginiamycin. Equine veterinary journal, v. 30, n. 2, p. 139-143, 1998. Disponível em:<https://doi.org/10.1111/j.2042-3306.1998.tb04473.x>. doi: 10.1111/j.2042-3306.1998.tb04473.x

JÚNIOR, A. C. Influência do tempo de estabulação no comportamnto de equinos da raça crioula . Unoesc \& Ciência-ACET, v. 6, n. 2, p. 201-208, 2015. 
KONIECZNIAK, P.; DIAS, I. F. T.; CALEFFO, T.; SINHORINI, W. A.; DO PRADO GUIRRO, E. C. B.. Estereotipias em equinos. Veterinária em Foco, v. 11, n. 2, p. 126-136, 2014.

KOOLHAAS, J. M., BARTOLOMUCCI, A.; BUWALDA, B. D.; DE BOER, S. F.; FLÜGGE, G.; KORTE, S. M.; ... RICHTER-LEVIN, G. Stress revisited: a critical evaluation of the stress concept. Neuroscience \& Biobehavioral Reviews, v. 35, n. 5, p. 1291-1301, 2011. Disponível em:< https://doi.org/10.1016/j.neubiorev.2011.02.003>. doi: 10.1016/j.neubiorev.2011.02.003

KUSANO, K.; HOBO, S.; ODE, H.; ISHIKAWA, Y. Tracheal endoscopic and cytological findings and blood examination results in thoroughbred racehorses suspected to have lower respiratory tract disease. Journal of equine science, v. 19, n. 4, p. 97-97, 2008. Disponível em:<https://doi.org/10.1294/jes.19.97>. doi: $10.1294 /$ jes.19.97

LEAL, B. B. Avaliação do bem-estar dos equinos de cavalaria da Polícia Militar de Minas Gerais: Indicadores etológicos, endocrinológicos e incidência de cólica. Belo Horizonte: UFMG, 2007. 61 p. Dissertação, Escola de Veterinária da Universidade Federal de Minas Gerais, Belo Horizonte, 2007.

LEWIS, L. D. Alimentação e cuidados do cavalo. São Paulo: Livraria ROCA, 1985. $248 \mathrm{p}$.

LEWIS, L. D. Nutrição clínica eqüina:alimentação e cuidados. São Paulo, Ed. Roca, 2000. 710p.

MAIR, T. S. Small intestinal obstruction caused by a mass of feedblock containing molasses in four horses. Equine Veterinary Journal, v. 34, n. 5, p. 532-536, 2002.2 Disponível em:<https://doi.org/10.2746/042516402776117719>. doi: $10.2746 / 042516402776117719$

MALINOWSKI, K. The horse industry's responsibility to animal welfare. 2004. Disponível em:<https://esc.rutgers.edu/fact_sheet/the-horse-industrysresponsibility-to-animal-welfare/>.

MARCELLA, K. L. Common behavior problems in horses. Equine Practice., v. 10, n. 6, p. 22-26, 1988.

MCGOWAN, T. W.; PINCHBECK, G.; PHILLIPS, C. J. C.; PERKINS, N.; HODGSON, D. R.; MCGOWAN, C. M. A survey of aged horses in Queensland, Australia. Part 2: clinical signs and owners' perceptions of health and welfare. Australian Veterinary Journal, v. 88, n. 12, p. 465-471, 2010. Disponível em:<https://doi.org/10.1111/j.1751-0813.2010.00638.x>. doi: 10.1111/j.1751-0813.2010.00638.x 
MCGREEVY, P. D.; CRIPPS, P. J.; FRENCH, N. P.; GREEN, L. E.; NICOL, C. J. Management factors associated with stereotypic and redirected behaviour in the Thoroughbred horse. Equine veterinary journal, v. 27, n. 2, p. 86-91, 1995. Disponível em:<https://doi.org/10.1111/j.20423306.1995.tb03041.x>. doi: 10.1111/j.2042-3306.1995.tb03041.x

MCGREEVY, P. Equine behavior: a guide for veterinarians and equine scientists. Londres: Saunders, 2004. 357p.

MCGREEVY, P. D.; MCLEAN, A. N.; WARREN-SMITH, A. K.; WARAN, N.; GOODWIN, D. Defining the terms and processes associated with equitation. Proceedings of the 1st International Equitation Science Symposium, August 2005. Broadford, Victoria, Post-Graduate Foundation in Veterinary Science, Sydney, 10-43.

MEDICA, P.; GIACOPPO, E.; FAZIO, E.; AVENI, F.; PELLIZZOTTO, R.; FERLAZZO, A. Cortisol and haematochemical variables of horses during a two day trekking event: effects of preliminary transport. Equine Veterinary Journal, v. 42, n. s38, p. 167-170, 2010. Disponível em:<https://doi.org/10.1111/j.2042-3306.2010.00197.x>. doi: 10.1111/j.20423306.2010.00197.x

$\mathrm{MENCH}$, J. A. Assessing welfare: an overview. Journal of Agricultural and Environmental Ethics, v. 6, n. 2, p. 68-75, 1993.

MILLS, D. S.; NANKERVIS, K. Comportamento eqüino. Roca: São Paulo, p. 213, 2005.

MOBERG, G. P. Problems in defining stress and distress in animals. Journal of the American Veterinary Medical Association, v. 191, n. 10, p. 1207, 1987.

MORRIS, E. A.; SEEHERMAN, H. J. Evaluation of upper respiratory tract function during strenuous exercise in racehorses. Journal of the American Veterinary Medical Association, v. 196, n. 3, p. 431-438, 1990.

MÖSTL, E.; PALME, R. Hormones as indicators of stress. Domestic animal endocrinology, v. 23, n. 1-2, p. 67-74, 2002. Disponível em:<https://doi.org/10.1016/S0739-7240(02)00146-7>. doi: 10.1016/S07397240(02)00146-7

MURRAY, M. J., GRODINSKY, C.; ANDERSON, C. W.; RADUE, P. F.; SCHMIDT, G. R. Gastric ulcers in horses: a comparison of endoscopic findings in horses with and without clinical signs. Equine Veterinary Journal, v. 21 , n. S7, p. 68-72, 1989. Disponível em:<https://doi.org/10.1111/j.20423306.1989.tb05659.x>. doi: 10.1111/j.2042-3306.1989.tb05659.x

NATIONAL RESEARCH COUNCIL - NRC. Nutrients requirements of domestic horses. 6.ed. Washington, D.C.: National Academy of Science, 
2007. 341p.

PALMA-DALLA, G.; CERRI, F.; POLES, C.; BARIANI, M. Úlcera gástrica em eqüinos. Revista Científica Eletrônica de Medicina Veterinária, Garça/FAMED, n. 8, 2007.

PARKER, M.; GOODWIN, D.; REDHEAD, E. S. Survey of breeders' management of horses in Europe, North America and Australia: comparison of factors associated with the development of abnormal behaviour. Applied Animal Behaviour Science, v. 114, n. 1, p. 206-215, 2008. Disponível em:<https://doi.org/10.1016/j.applanim.2008.02.003>. doi: 10.1016/j.applanim.2008.02.003

PASCOE, J. R. Exercise induced pulmonary haemorrhage. In: Beech, J. Equine Respiratory Disorder. Pensilvania: Lea \& Febiger, 1991.

PEETERS, M.; SULON, J.; BECKERS, J. F.; LEDOUX, D.; VANDENHEEDE, M. Comparison between blood serum and salivary cortisol concentrations in horses using an adrenocorticotropic hormone challenge. Equine veterinary journal, v. 43, n. 4, p. 487-493, 2011. Disponível em:<https://doi.org/10.1111/j.2042-3306.2010.00294.x>. doi: 10.1111/j.20423306.2010.00294.x

PESSOA, F. N.; PRADO, C. C. Larousse dos Cavalos, 1a ed. São Paulo: Larousse do Brasil, 2006. $288 \mathrm{p}$.

PULZ, R. S. Ética e bem-estar animal. Canoas: Editora Ulbra, v. 1, 2013.

REEF, V. B. Stress echocardiography and its role in performance assessment. Veterinary Clinics: Equine Practice, v. 17, n. 1, p. 179-189, 2001.

REZENDE, M. J. D. M., PIMENTEL, C. M. M., PALUDO, G. R., MARTINS, R. D., OLIVEIRA, L. D. P. G. D., FUCK, B. H., \& LOUVANDINI, H. Comportamento de cavalos das raças Bretã e Percheron estabulados. Ciência Animal Brasileira, v. 7, p. 17-25, 2006a.

REZENDE, M. J., MCMANUS, C., MARTINS, R. D., DE OLIVEIRA, L. D. P. G., GARCIA, J. A. S., \& LOUVANDINI, H. Comportamento de cavalos estabulados do exército brasileiro em Brasília. Ciência Animal Brasileira, v. 7, n. 3, p. 327-337, 2006b.

RIBEIRO, G.; SILVA, L. C. L. C. D.; BELLI, C. B.; VARGAS, L. P.; PIFFER, M. L. T.; MIRIAN, M.; FERNANDES, W. R. Occurrence of gastric ulcers in horses exercised on a treadmill. Ciência Rural, v. 46, n. 5, p. 909-914, 2016. Disponível em:<http://dx.doi.org/10.1590/0103-8478cr20150856>. doi: $10.1590 / 0103-8478 c r 20150856$ 
RIBEIRO, L. B.; Furtado, C. E.; Tonello, C. L.; Barbosa, E. D.; Brandi, R. A. Comportamento e distúrbios alimentares em eqüinos durante ensaio de metabolismo recebendo volumosos com diferente qualidade nutricional acrescido de probiotico (saccharomyces cerevisiae). Revista da FZVA, v. 16, n. 1, 2009.

ROBERTS, M. O homem que ouve cavalos. Bertrand Brasil, 2002.

ROSS, M. W.; DYSON, S. J. Diagnosis and Management of Lameness in the Horse. Saunders, Philadelphia, PA, 2003.

SALTER, R. E.; HUDSON, R. J. Feeding ecology of feral horses in western Alberta. Journal of Range Management, p. 221-225, 1979. DOI: $10.2307 / 3897127$

SCHMIDT, A.; AURICH, J.; MÖSTL, E.; MÜLLER, J.; AURICH, C. . Changes in cortisol release and heart rate and heart rate variability during the initial training of 3-year-old sport horses. Hormones and Behavior, v. 58, n. 4, p. 628-636, 2010. Disponível em:<https://doi.org/10.1016/j.yhbeh.2010.06.011>. doi: 10.1016/j.yhbeh.2010.06.011

SILVA, C. O cavalo atleta. Revista Veterinária Actual. p. 18-22. 2010.

SILVA, E. L. Revisão para embasar o desenvolvimento de ferramenta prática para avaliação do bem-estar de cavalos com base em indicadores físicos e mentais. 2014. 62f. Trabalho de Conclusão de Curso (graduação em Zootecnia) - Universidade Federal de Santa Catarina. Centro de Ciências Agrárias, Florianópolis, SC.

SMIET, E.; VAN DIERENDONCK, M. C.; SLEUTJENS, J.; MENHEERE, P. P. C. A.; VAN BREDA, E.; DE BOER, D.; VAN DER KOLK, J. H. Effect of different head and neck positions on behaviour, heart rate variability and cortisol levels in lunged Royal Dutch Sport horses. The Veterinary Journal, v. 202, $\quad$ n. $1, \quad$ p. 26-32, $2014 . \quad$ Disponível em:<https://doi.org/10.1016/j.tvjl.2014.07.005>. doi: 10.1016/j.tvjl.2014.07.005

SMYTHE, R. H. A psique do cavalo. São Paulo: Internacional DATA S.A., 1990. $141 \mathrm{p}$.

SNOWDON, C. T. O significado da pesquisa em comportamento animal. Estudos de Psicologia (Natal), v. 4, n. 2, p. 365-373, 1999. Disponível em:<http://dx.doi.org/10.1590/S1413-294X1999000200011>. doi: 10.1590/S1413-294X1999000200011

SOBESTIANSKY, J.; ZANELLA, J. R. C. Formas anormais de comportamento. Doenças dos suínos. Goiânia: Cânone Editorial, p. 579592, 2007.

STASHAK, T. S. Adam's lameness in the horses. 5 ed. Philadelphia: ENCICLOPÉDIA BIOSFERA, Centro Científico Conhecer - Goiânia, v.15 n.27; p. 225 
Lippincott Williams \& Wilkins. 1998. p. 645-1037.

SUMA, R. P. et al. Hemorragia pulmonar induzida pelo exercício em cavalos da raça puro sangue inglês. Revista Brasileira de Ciência Veterinária, Supl., v.9, n.1, p. 104-05, 2002.

THOMASSIAN, A. Enfermidades dos Cavalos, editora Varela. São Paulo, p. 265, 2005

THORNE J. B.; GOODWIN, D.; KENNEDY, M. J.; DAVIDSON, H. P. B.; HARRIS, P. Foraging enrichment for individually housed horses: Practicality and effects on behavior. Applied Animal Behaviour Science, v. 94, n. 1-2, p. 149-164, 2005.

Disponível em:<https://doi.org/10.1016/j.applanim.2005.02.002>. doi: 10.1016/j.applanim.2005.02.002

TURNER, T. A.; ADAMS, S. B.; WHITE, N. A. Small intestine incarceration through the epiploic foramen of the horse. Journal of the American Veterinary Medicine Association. v. 184, n. 6, p. 731-734, 1984.

VACHON, A. M.; FISCHER, A. T. Small intestinal herniation through the epiploic foramen: 53 cases (1987-1993). Equine Veterinary Journal, v. 27, n. 5, p. 373-380, 1995. Disponível em:<https://doi.org/10.1111/j.20423306.1995.tb04073.x>. doi: 10.1111/j.2042-3306.1995.tb04073.x

VERVUERT, I.; COENEN, M. Nutritional management in horses: selected aspects to gastrointestinal disturbances and geriatric horses. In: Proceedings of the 2nd European Equine Nutrition and Health Congress. 2004. p. 1920.

VIEIRA, M. C. Percepções e práticas de manejo em estabelecimentos equestres quanto à influência dessas práticas para o bem-estar de equinos. 2015. 100 p. Dissertação (Mestrado em Agroecossistemas). Universidade Federal de Santa Catarina, 2015.

VON BORSTEL, U. U.; DUNCAN, I. J. H.; SHOVELLER, A. K.; MERKIES, K.; KEELING, L. J.; MILLMAN, S. T. Impact of riding in a coercively obtained Rollkur posture on welfare and fear of performance horses. Applied Animal Behaviour Science, v. 116, n. 2, p. 228-236, 2009.

WATERS, F. J.; NICOL, C. J.; FRENCH, N. P. Factors influencing the development of stereotypic and redirected behaviours in young horses: findings of a four year prospective epidemiological study. Equine veterinary journal, v. 34, n. 6, p. 572-579, 2002. Disponível em:< https://doi.org/10.2746/042516402776180241>.

doi: $10.2746 / 042516402776180241$

WIEMER, P.; GRUYS, E.; VAN HOECK, B. A study of seven different types of grafts for jugular vein transplantation in the horse. Research in veterinary science, v. 79, n. 3, p. 211-217, 2005. Disponível em:< ENCICLOPÉDIA BIOSFERA, Centro Científico Conhecer - Goiânia, v.15 n.27; p. 226 
https://doi.org/10.1016/j.rvsc.2004.12.005>. doi: 10.1016/j.rvsc.2004.12.005

WILLIAMS, L. R.; WARREN-SMITH, A. K. Conflict responses exhibited by dressage horses during competition. Journal of Veterinary Behavior: Clinical Applications and Research, v. 5, n. 4, p. 215, 2010. Disponível em:<https://doi.org/10.1016/j.jveb.2009.11.002>.

doi:

10.1016/j.jveb.2009.11.002

ZANELLA, A. J. Indicadores fisiológicos e comportamentais do bem-estar animal. In: Conferencia Internacional: Tecnología e Desenvolvimento Sustentavel 18-22 Set 1995 Porto Alegre (Brasil). UFRG (Brasil) EMBRAPA (Brasil), 1995.

ZANELLA, A. J. Fatores que põem em risco o bem-estar de suínos ao ar livre. Simpósio sobre sistema intensivo de suinos criados ao ar livresiscal , v. 1, p. 157-167, 1996. 